\title{
Construction and Model of Index System under Information Condition
}

\section{Linlin Li}

Xi'an Research Inst. of High-tech

Xi'an, China, 710025

E-mail: Iilinlin74@yeah. net

\section{Yunfei Lu}

Xi'an Research Inst. of High-tech

Xi'an, China, 710025

E-mail: $2501470556 @$ @q. com

\section{Zhuang Zhang}

Xi'an Research Inst. of High-tech

Xi'an, China, 710025

E-mail: $3207969734 @$ @q. com

\begin{abstract}
With the development of information technology, the role of information superiority in combat command is becoming more and more prominent, but the traditional index system can not meet the system evaluation under the informationization condition; therefore, based on the characteristics of operational command and control system and the traditional index system, this paper integrates the information advantage with the evaluation index system to construct a more realistic evaluation index system. In addition, on the basis of reading a large number of references and in combination with the actual system, a quantifiable mathematical model was established for each evaluation index to sum up the three points in index system construction process. Finally, the correctness of the index system is verified by simulation experiment.
\end{abstract}




\section{Introduction}

The information superiority [1] is defined as "the ability of continuously collecting, processing and distributing information while preventing the enemy from doing the same thing". With the network center war[2] put forward, the original independent command, communication, intelligence, combat nodes are connected with a close overall. The central purpose of the network center war and the Global Information Grid (GIG) proposed by the US military is to gain the absolute information advantage on a global scale. In order to adapt to the new military reform, obtain the absolute information superiority and the information right[3], the research on the command and control system based on the information superiority has gradually become the hot spot of attention.

At present, there have been many researches on the index system of command and control system in domestic military field. Although the results are relatively abundant, the information superiority included as an evaluation index is relatively small. Based on the new information of information technology, this paper constructs the index system of command and control system based on information superiority, and establishes the quantifiable mathematical model according to the characteristics of each index.

\section{Construction of Index System Based on Information Superiority}

The construction of the index system is a systematic project, which is directly related to the evaluation efficiency of the system and needs to fully reflect the system characteristics. This section summarizes the basic principles followed in the construction of the index system and constructs the index system based on the information superiority.

\subsection{Index System Construction Principle}

The construction of the index system should select the representative key indicators to avoid redundant indicators and model complexity, ensure simple and efficient system evaluation according to the principles[4-6] as follows:

1) The simplest principle: the selection of indicators must be able of objectively reflecting the basic characteristics of the command and control system, as little as possible to evaluate the key system performance.

2) The principle of comprehensiveness: the selected indicators must be able of describing the comprehensive characteristics of the system in all directions.

3) The testability principle: try to easily select the quantitative calculation of indicators to ensure the data reliability.

4) The stability principle: robustness, must ensure that the stability of the index system, the external factors have a certain degree of anti-interference ability.

5) The principle of timeliness: with the improvement of information degree, the battlefield situation is changing rapidly and the selection of indexes must be able of timely and accurately responding system changes with strong flexibility.

6) The principle of independence: the selection of indexes should avoid cross-overlap between indicators as much as posible, on the one hand to avoid the interaction between indicators, on the other hand to reduce the complexity of systematic evaluation.

\subsection{Index System Construction}


According to the above principles of establishing the index system, the evaluation index system based on the information superiority is established, as shown in Figure 1.

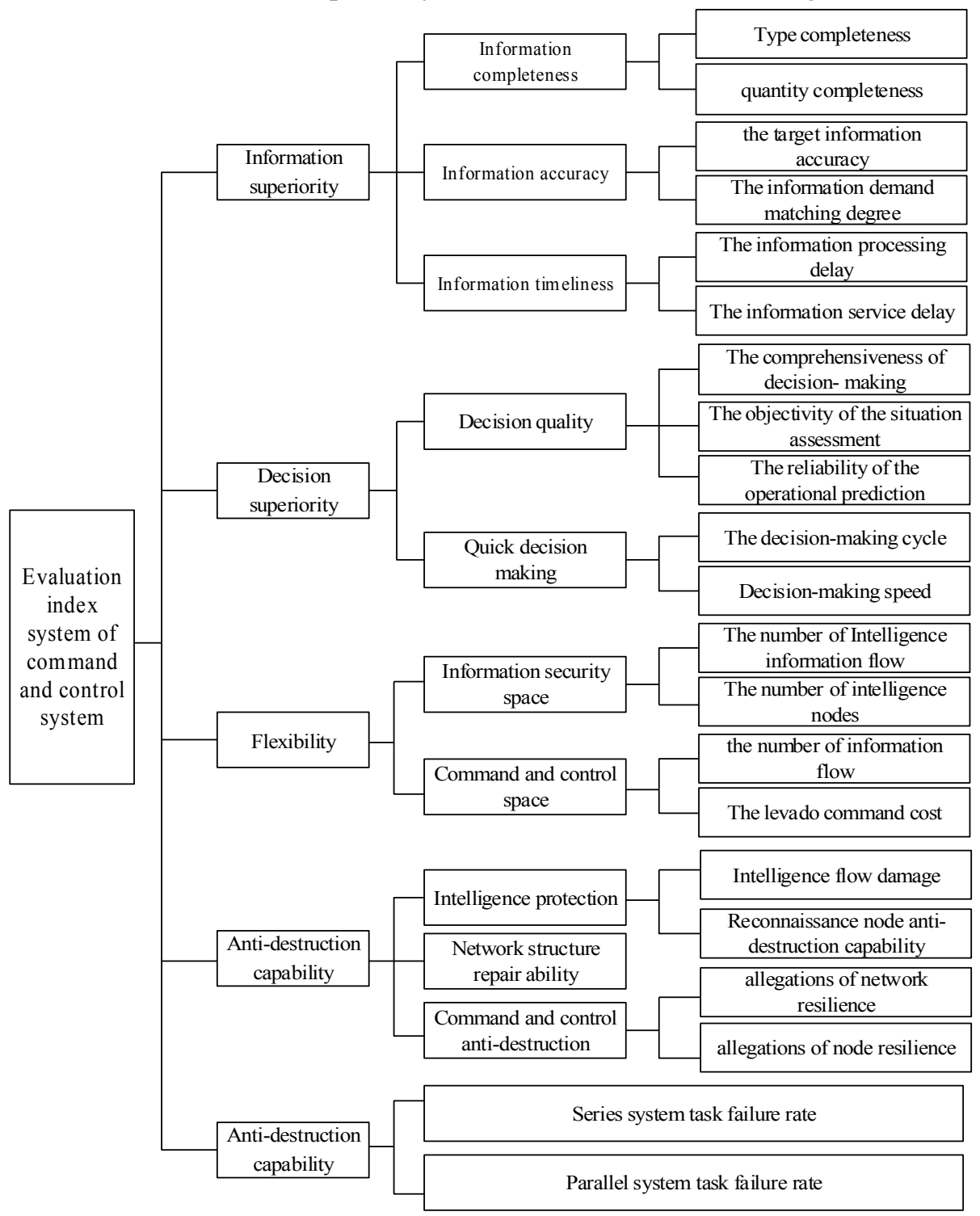

Figure 1: Evaluation Index System of Command and Control System based on Information Superiority

\section{Index Evaluation Model}

The system performance evaluation is like a chemical reaction, the evaluation index system is the reactant and the basis of assessment, and the mathematical model is the reaction conditions. In this section, based on the establishment of the evaluation index system, a typical mathematical model is established according to the characteristics of each index.

\subsection{Information Superiority}

1. Information completeness[1]refers to the battlefield situational awareness of the enemy's strategic objectives or the number of incoming targets and the number of objective actual target ratio, including type completeness $C(t)$ and quantity completeness $D(t)$. The 
calculation is as follows:

$$
C(t)=\rho(t) / \phi(t)
$$

$\phi(t)$ indicates the actual number of enemy targets in the objective situation at time $\mathrm{t} ; \rho(t)$ indicates the number of categories in which the enemy target has been correctly identified at the moment of perception.

$$
D(t)=\eta(t) / \lambda(t)
$$

$\lambda(t)$ indicates the actual number of enemy targets in the objective situation at time $t ; \eta(t)$ indicates the number of times that the enemy target has been found at the moment of perception.

The information completeness at time $\mathrm{t}$ is $F(t)$

$$
F(t)=C(t) \times D(t)
$$

2. The information accuracy is closely related to the degree of matching with the enemy target characteristics and the real target characteristics in situational awareness. As the matching of information can fulfill the decision-making needs, it can be measured from the target information accuracy and the information demand matching degree.

(1) The conceptual content of the target information accuracy can be measured by using the mean value of the feature parameters of each target in the distribution link. The formalized calculation model is as follows:

$$
\operatorname{Validity}(t)=\left(\operatorname{vali}_{s}(t) \mid \text { vali }_{o}(t), \text { vali }_{p}(t)\right)=\frac{\frac{\sum_{i=1}^{m} \sum_{j=1}^{n} g_{i j}-f_{i j}}{g_{i j}}}{m * n}
$$

$\operatorname{vali}_{O}(t), \operatorname{vali}_{P}(t)$ and $\operatorname{vali}_{S}(t)$ indicates collection, integration and distribution of three stages of information accuracy. $g_{i j}$ indicates the information state of the $j$ feature of the $i$ object provided by the distribution node; $f_{i j}$ indicates the information state of the $j$ feature of the $i$ target; $m$ indicates total number of targets; $n$ indicates the characteristic parameter length of the target。

(2) The information demand matching degree indicates the information satisfaction that the information processing node distributes the information to the usage node and these using nodes' satisfaction of demand information. It can be measured by the degree to which information is used by the node to respond to each type of information demand. Therefore, the formal calculation model of information demand matching is shown as follows:

$$
\text { Settle }=\sum_{i=1}^{n} \frac{p_{i}}{r_{i}}
$$

$p_{i}$ indicates the $i$ information provided by the information processing node to the information usage node; $r_{i}$ indicates the $i$ complete information required for the information usage node; $n$ indicates the total number of message types required for the information usage node.

3. The information timeliness can be expressed as the delay of information transmission from the reconnaissance node to the information usage node. And t can be measured from the information processing delay and the information service delay.

(1) The information processing delay can be expressed as the delay of the information 
transmission from the reconnaissance node to the information processing node. The formalized model of the information processing delay indicator is as follows:

$$
\operatorname{Delay}(t)_{t}=\operatorname{Max}\left(\sum_{u=1}^{w} \operatorname{delay}(t)_{i, u}+\sum_{c=1}^{v} \operatorname{delay}(t)_{i, c}\right)
$$

$\operatorname{delay}(t)_{i, u}$ indicates the processing delay of the $u$ node in the maximum delay; $\operatorname{delay}(t)_{i, c}$ indicates the transmission delay of the $c$ side of the maximum delay in the information flow; $w$ indicates the maximum number of nodes that the delay maximum information flow contains; $v$ indicates the number of links that the delay maximum information flow contains.

(2) The information service delay can be expressed as the information transmission delay of the information processing node to the information usage node, and the formalized model is as follows:

$$
\operatorname{Delay}(t)_{s}=\frac{\sum_{i=1}^{n}\left(\sum_{u=1}^{w} \operatorname{delay}(t)_{i, u}+\sum_{c=1}^{v} \operatorname{delay}(t)_{i, c}\right)}{n}
$$

$\operatorname{delay}(t)_{i, u}$ indicates the processing delay of the $u$ node of the $i$ information flow; $\operatorname{delay}(t)_{i, c}$ indicates the transmission delay in the $c$ side of the $i$ information flow; $w$ indicates the number of nodes contained in each stream; $v$ indicates the number of links contained in each stream; $n$ indicates the total number of information flows.

And the final information timeliness is the sum of the information processing delay and the information service delay, and the index formal model is as follows:

$$
\operatorname{Delay}(t)_{\mathrm{inf} o r}=\operatorname{Delay}(t)_{t}+\operatorname{Delay}(t)_{s}
$$

\subsection{Decision Superiority}

1. The decision quality is mainly related to the comprehensiveness of decision-making, the objectivity of situation assessment and the reliability of operational prediction.

(1) The comprehensiveness of decision-making is mainly reflected in the decision-making support elements of the comprehensive aspects of the decision:

$$
\text { overrall }=\sum_{i=1}^{n} \frac{N_{i}-\text { give }}{N_{i}-\text { need }}
$$

$N_{i-\text { give }}$ and $N_{i-n e e d}$ are the number of decision factors that can be provided by the decision-making nodes in the system and the number of decision support elements needed for decision-making.

(2)The objectivity of the situation assessment is mainly reflected in the consistency of the battlefield situation assessment and objective facts, measured by the completeness $F(t)$ and accuracy $V(t)$ of the situational perceived quality, and the results is same as the results in 2.1.

(3) The reliability of operational prediction can be predicted by the commander's loss to the actual loss and the proportion of the successful blow. the value normalized is expressed as:

$$
R=\frac{\min (\text { predicted loss, actual loss })}{\max (\text { predicted loss, actual loss })}
$$


the value of predicted loss and actual loss can be calculated by the predicted and actual combat consumption of human, material, financial and so on.

At the same time, the proportion of successful strike is expressed as:

$$
W=\frac{\operatorname{laun}_{\text {suc }}}{\text { laun }_{\text {all }}}
$$

laun $_{\text {suc }}$ and laun $_{\text {all }}$ indicates the number of successful attacks and the total number of blows.

2. Decision-making speed can be reflected in the decision-making cycle and decisionmaking speed.

(1)The decision-making cycle $t_{D}$ is composed of a combination of battlefield situation assessment time $t_{C}$, forecasted operational planning time $t_{R}$, combat action time $t_{A}$, and result feedback time $t_{B}$.

$$
t_{D}=t_{C}+t_{R}+t_{A}+t_{B}
$$

The decision-making process, which includes the battlefield situation assessment time $t_{C}$, the combat planning time $t_{R}$, the command time $t_{M}$, the weapon reaction time $t_{W}$, the combat time $t_{F}$ and other influential factors. For each decision-making process, only when the sum of the time is less than the battlefield warning time $t_{S}$, or less than the corresponding time of the enemy, the decision-making highlights the time advantage.

$$
t_{D}=t_{C}+t_{R}+t_{M}+t_{F}<t_{S}
$$

$t_{M}$ indicates the time that all levels of command from the beginning of the command to command the execution report; $t_{W}$ indicates the time required for the weapon system to receive the strike order from the beginning of the strike; $t_{F}$ indicates the time required for the weapon system to complete from the blow to the strike.

(2) Decision-making speed: if the commander can accurately grasp the favorable situation and reliably select the action process, get faster than the opponent's decision-making speed, it will occupy a favorable position in the battle. The decision-making speed is defined as:

$$
D_{v}=(F(t) \times V(t)) /\left(t_{C}+t_{R}+t_{A}+t_{B}\right)
$$

In practical applications, the value $D_{V}$ is the average of multiple decisions.

\subsection{Flexibility}

Flexibility is a measure of the system's ability of adapting to the internal and external environmental changes. It can be measured from the difference of the system structure and the cost. It mainly includes two aspects: the information security space and the command and control space.

1.The information security space can also be understood as the diversity of information services, including the number of intelligence information flow and the intelligence information source node number.

(1) The number of intelligence information flow refers to the information security information transmission path. The more information transmission path and intelligence 
protection are, the greater the space for change and the better the flexibility will be.

(2) The number of intelligence nodes is the number of information centers that provide information services. The more the number and the more diversified the way of information services are, the better the flexibility will be.

2.The command and control space is a measure of the system's ability of structure command control to adapt itself to the internal and external changes, including the number of information flow and the levado command cost.

(1) The number of information flow: combat the node as the end weapon node in the system, its command mode is the most, the command is also the most flexible.

(2) The levado command cost: with more the more commanders, the more the number of allegations, but not the more steers the better, because any kind of leapfrog command is the cost of the cost of the system to meet the constraints of the number of allegations, the cost of the cost The higher the higher.

\subsection{Anti-destruction Capability}

Anti-destruction capability is the ability of maintaining the original performance when the relationship between the system node and the node is uncertain. Including the protection of information security capabilities, network structure repair capacity, command and control antidestruction capability.

1.Intelligence protection is mainly to serve the command and decision mainly by two factors. It must firstly be able of detecting the detection of intelligence information and secondly it must be able of successfully transmiting intelligence information to the command node, to protect the information security, including intelligence information flow damage and reconnaissance node destruction capability.

(1)Intelligence flow damage refers to the information transmission link upon completion of information push under the premise of "broken" and "damage" to the maximum.

Therefore, if the system structure $G$ is attacked, the damage to the information flow $\varepsilon_{G^{\prime}}$ can be measured as follows:

$$
\varepsilon_{G^{\prime}}=\frac{N u m_{\text {infor }}}{\operatorname{Sum}_{\text {inf } o r}}
$$

$N_{u m} m_{\text {inf or }}$ indicates the number of information flow after the attack. Sum $m_{\text {inf } o r}$ indicates the total number of intelligence flows in the original structure.

(2)Reconnaissance node anti-destruction capability: the reconnaissance node is the prerequisite for the transmission of intelligence information; therefore, the anti-destruction capability of the reconnaissance node can not be neglected in the system survivability assessment and the survivability of the node can be measured by the reconfiguration of the reconnaissance node.

2.Command and control the anti-destruction capability: command and control of the antidestruction capabilities include allegations of network resilience and allegations of node resilience, and its computational model is similar to the intelligence-protected resilience, which will not be described here.

3.Network structure repair ability: the network structure repair ability refers to the ability that troops repair the network damaged to normal and can complete the normal completion of the task. Generally, the network structure repair ability can be described by the probability of 
repair success.

$$
E(t)=\left\{\begin{array}{l}
\frac{\text { time }}{\text { sumtime }}, \text { success } \\
0, \text { unsuccess }
\end{array}\right.
$$

time indicates the actual time of completing the repair; sumtime indicates that the task can be successfully completed to allow the longest time.

\subsection{Anti-destruction Capability}

The reliability index is divided into the series system's task failure rate and the parallel system task failure rate. It can reflect the reliability of system command and control to calculate the indicators, one by one as follows.

1. Series system task failure rate is composed of $n$ nodes composed of a series system, in which any node failure will lead to the failure of the entire system, that is, the use of the system node failure rate to calculate the sum of the model can be expressed as:

$$
\lambda_{\text {ser }}=\sum_{i=1}^{n} \lambda_{i}
$$

$\lambda_{i}$ Indicates the probability that the $i$ node has failed.

2.The parallel system task failure rate is defined by the $n$ nodes of the parallel system. When one of the nodes work properly, the system is working properly; when all units fail, the system is invalid, that is, the use of the system node failure rate accumulation Calculation, the calculation model can be expressed as:

$$
\lambda_{\text {par }}=\sum_{i=1}^{n} \lambda_{i}
$$

$\lambda_{i}$ indicates the probability that the $i$ node has failed.

\section{Experiment and Analysis}

\subsection{Main Points of the Index System Should Be Grasped}

Whether the evaluation index system is comprehensive, objective and concise will exert direct impact on the final assessment results, and construct a set of indicators that can effectively respond to the characteristics of the system. The evaluation results are basically realistic[7-9]in addition to the above principles. Focus on the following points:

(1)Evaluate the quantification of indicators. In order to ensure the systematic assessment is reasonable and efficient, the evaluation index system must be used in the top-down and step-bystep decomposition method until all the evaluation indicators can be quantified.

(2)Highlight the importance of information superiority. With the improvement of informationization degree, the right of information has gradually become the key factor to obtain the winning power. The information superiority ability directly determines the command and decision ability of the command and control system of the enemy and the enemy as well as the relationship between the battlefield and the whole battlefield to a certain extent. When assessing the index system, the information superiority must be included as an important factor in the index system.

(3)Focus on the integration of assessment systems and allegations. At present, all the 
military system of assessment system and the allegation system are constructed independently. When such a system assessment and construction ideas can adapt themselves to the new situation under the military struggle to prepare the request is still open to question. This paper argues that under the current situation, the command and control system and the evaluation system should pay attention to the integration construction. The battlefield situation is changing rapidly,therefore the system assessment can not be fully evaluated in advance. Instead, the command and control system can be dynamic evaluated on the battlefield in real time with highly flexible. This system not only features highly reliable and efficient command and control capabilities, but also have a flexible and efficient assessment capabilities.

\subsection{Experiment and Simulation}

This section of the simulation experiment is running under Microsoft Windows7 operating system, the basic configuration: Intel CoreTM i7 CPU, 4G memory, the use of matlab2012 simulation. The validity of the evaluation system is based on the cloud model-based effectiveness evaluation method, the comprehensive fuzzy evaluation method[11] and the cloud center of gravity judgment method[12].

Step 1 Index weight

\begin{tabular}{ccccccccccccccccccccccccc}
\hline Index & U111 & U112 & U121 & U122 & U131 & U132 & U211 & U212 & U213 & U221 & U222 & U311 & U312 & U321 & U322 & U411 & U412 & U431 & U432 \\
\hline Grade & 1 & 2 & 1 & 2 & 1 & 1 & 2 & 1 & 3 & 2 & 1 & 1 & 2 & 1 & 2 & 2 & 1 & 1 & 2 \\
$W_{i}$ & 1 & 0.5 & 1 & 0.5 & 1 & 1 & 0.65 & 1 & 0.35 & 0.5 & 1 & 1 & 0.5 & 1 & 0.5 & 0.5 & 1 & 1 & 0.5 \\
$W_{\mathrm{i}}^{*}$ & 0.67 & 0.33 & 0.67 & 0.33 & 0.5 & 0.5 & 0.33 & 0.5 & 0.17 & 0.33 & 0.67 & 0.67 & 0.33 & 0.67 & 0.33 & 0.33 & 0.67 & 0.67 & 0.33 \\
\hline
\end{tabular}

Table 1: Weight of the Three Indicators

\begin{tabular}{ccccccccccccc}
\hline Index & U11 & U12 & U13 & U21 & U22 & U31 & U32 & U41 & U42 & U43 & U51 & U52 \\
\hline Grade & 2 & 1 & 1 & 1 & 2 & 1 & 1 & 2 & 1 & 3 & 1 & 1 \\
$W_{i}$ & 0.65 & 1 & 1 & 1 & 0.5 & 1 & 1 & 0.65 & 1 & 0.35 & 1 & 1 \\
$W_{i}^{*}$ & 0.24 & 0.38 & 0.38 & 0.67 & 0.33 & 0.5 & 0.5 & 0.33 & 0.5 & 0.17 & 0.5 & 0.5 \\
\hline
\end{tabular}

Table 2: Weight of the Secondary Inde

\begin{tabular}{cccccc}
\hline Index & U1 & U2 & U3 & U4 & U5 \\
\hline Grade & 2 & 1 & 3 & 4 & 3 \\
$\mathrm{~W}_{\mathrm{i}}$ & 0. 73 & 1 & 0. 61 & 0.39 & 0.61 \\
$\mathrm{~W}_{\mathrm{i}}{ }^{*}$ & 0. 22 & 0. 30 & 0. 18 & 0. 12 & 0.18 \\
\hline
\end{tabular}

Table 3: Weight of the Primary Index

\section{Step 2 Index Value}




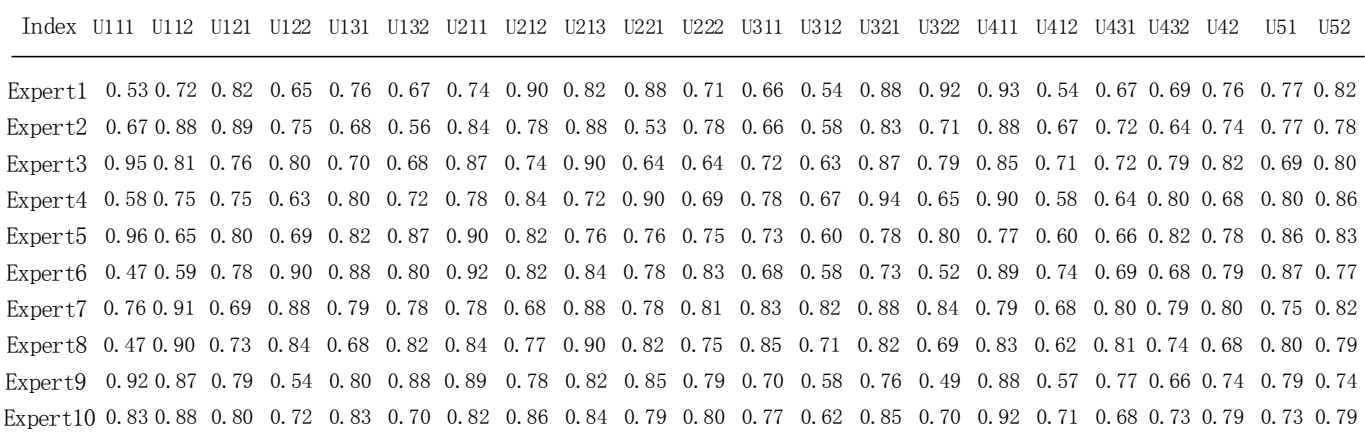

Table 4: Evaluation Value of Each Index

Step 3 Comprehensive Evaluation Results

\begin{tabular}{ccccc}
\hline $\begin{array}{c}\text { Evaluation } \\
\text { grade }\end{array}$ & Poor & Medium & Good & Excellent \\
\hline Interval division & {$[0,0.50]$} & {$[0.35,0.85]$} & {$[0.60,0.98]$} & {$[0.80,1]$} \\
$\begin{array}{c}\text { Cloud model } \\
\text { assessment }\end{array}$ & $1.312 \times 10^{-4}$ & 0.193 & 0.640 & 0.083 \\
$\begin{array}{c}\text { Fuzzy comprehensive } \\
\text { assessment }\end{array}$ & 0.008 & 0.204 & 0.656 & 0.132 \\
$\begin{array}{c}\text { Cloud center of gravity } \\
\text { judgment }\end{array}$ & & 0.712 & \\
\hline
\end{tabular}

Table 5: Evaluation Result

From the comparison results of Table 5, the result by the three evaluation methods is the same and the evaluation results are good, indicating that the index system is feasible.

\section{Conclusion}

Under the informationization condition, the information superiority has become an indispensable combat factor. It is very important to measure the information advantage effectively and incorporate it into the system application efficiency in the system comprehensive performance evaluation. Based on the characteristics of a command and control system of a weapon and equipment, this paper constructs a complete system evaluation index system, integrates the information advantage into the comprehensive performance evaluation and establishes the corresponding mathematical model according to the characteristics of each index. Finally, In the prone to a few questions, key points are put forward. It is simple and effective to construct a reasonable, comprehensive and established mathematical model based on the information and control system of the command and control system based on the information superiority. However, due to the slow progress of the research, the integration between the indexes and comprehensive efficiency assessment method has not yet been mature but in request of further study.

\section{References}

[1] JIANG Han, YIN Hao, LI Xue-jun, CAO Ke-jing. Measure of information superiority in simulation of $C^{4} I S R$ gaming[J]. Journal of Systems Engineering and Electronics. 2006, 28(1):88-91. (In Chinese) 
[2] DAVID, JOHN J G, FREDERICK P S. Network centric warfare: developing and leveraging information superiority[J]. DoD C4ISR Cooperative Research Program, 1999.

[3] XU Chang, LI Lu-ji. Research on Information Superiority Impose on Operation[J] . Ship Electronic Engineering. 2011,31(1):95-99. (In Chinese)

[4] YU Li, YUE Zhen-jun, LIANG Kun-tai. Construction of military intelligence effectiveness evaluation index system [J]. Journal of Intelligence. 2010, 29(12):116-118. (In Chinese)

[5] QI Zongfeng, HAN Shan, LI Jianxun. Applications of Generalized Rough Set Theory in Evaluation Index System of Radar Anti-Jamming Performance[J]. Shanghai Jiaotong Univ. (Sci.), 2016, 21(2):151-1.58.

[6] Jin-shan Yang, Feng-you Wang. Developing a quantitative index system for assessing sustainable forestry management in Heilongjiang Province, China: a case study[J]. CrossMark, 2016, 27(3):611-619.

[7] CUI Wen-xiong, DAI Tong-hui, ZHANG Guo-wei. Research on Effectiveness Evaluation of Network Combat[J]. Ordnance Industry Automation. 2009,28(5): 14-15. (In Chinese)

[8] WANG Min-le, PENG Si-ping, YANG Xian-de, LI Peng-fei. Missile weapon system survivability analysis method[M]. National Defence Industry Press. 2015. (In Chinese)

[9] LI Xiang-yang, PAN Chang-peng, LI Wei-bo. Index Syetem of Combat Effectiveness of Command \& Control System Based on C-AHP[J]. Ship Electronic Engineering. 2014, 34(2):31-34. (In Chinese)

[10] FANG Jian, WANG Yue, YUAN Jian. Measurement of information superiority based on set distance[J]. Systems Engineering and Electronics. 2017,39(1):114-119. (In Chinese)

[11] CHEN Zhao-bing, GUO Jin, WANG Bing. Operational efficiency evaluation of vehicle carrying and high supporting optic-electronic detecting system $[\mathrm{J}]$. Optics and Precision Engineering. 2013,21(1):77-86. (In Chinese)

[12] YANG Feng, WANG Bi-yao, ZHAO Hui-bo, WU Jun. Effectiveness evaluation for strategy early-warning information system based on cloud model[J]. Systems Engineering and Electronics. 2014,36(7):1334-1338. (In Chinese) 